\title{
Effect of urinary angiotensinogen and high-salt diet on blood pressure in patients with chronic kidney disease: results from the Korean Cohort Study for Outcome in Patients with Chronic Kidney Disease (KNOW-CKD)
}

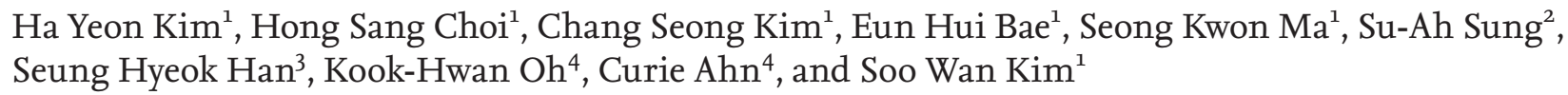

\begin{abstract}
${ }^{1}$ Department of Internal Medicine, Chonnam National University Medical School, Gwangju; ${ }^{2}$ Department of Internal Medicine, Nowon Eulji Medical Center, Eulji University, Seoul; ${ }^{3}$ Department of Internal Medicine and Institute of Kidney Disease Research, Yonsei University College of Medicine, Seoul; ${ }^{4}$ Department of Internal Medicine, Seoul National University College of Medicine, Seoul, Korea
\end{abstract}

Received: February 27, 2020

Revised : April 10, 2020

Accepted: April 23, 2020

\section{Correspondence to}

Soo Wan Kim, M.D.

Department of Internal

Medicine, Chonnam National

University Medical School, 42

Jebong-ro, Dong-gu, Gwangju

61469 , Korea

Tel: $+82-62-220-6271$,

Fax: $+82-62-225-8578$

E-mail:skimw@chonnam.ac.kr https://orcid.org/0000-0002-

3540-9004
Background/Aims: This study aimed to investigate whether urinary angiotensinogen (UAGT) excretion was associated with elevated blood pressure in patients with chronic kidney disease (CKD) and to evaluate the relationship among blood pressure, intra-renal renin-angiotensin system (RAS) activity, and dietary sodium in patients with CKD.

Methods: Participants from the Korean Cohort Study for Outcome in Patients with Chronic Kidney Disease (KNOW-CKD) were included. Of the total cohort of 2,238 individuals with CKD, we included 1,955 participants who underwent complete 24-hour urinary sodium (24-hour UNa) analysis. They were categorized into three groups according to three tertiles of their 24-hour UNa, reflecting daily salt intake. To measure intra-renal RAS activity, the UAGT excretion was assayed with an enzyme-linked immunosorbent assay.

Results: Elevated 24-hour UNa levels, logarithm of UAGT-to-creatinine ratio (UAGT/Cr), increased waist-to-hip ratio, and decreased estimated glomerular filtration rate were the risk factors for increased systolic blood pressure. Systolic blood pressure showed a positive correlation with 24-hour UNa levels and logarithm of UAGT/Cr.

Conclusions: UAGT and urinary sodium excretion are independent determinants of systolic blood pressure in patients with CKD. These findings suggest that increased systolic blood pressure in CKD patients is associated with both increased dietary sodium levels and intra-renal RAS activity. The risk of elevated systolic blood pressure in the 3 rd tertile of both the UAGT/Cr and 24-hour UNa groups was about 2.3 times higher than that in the reference group.

Keywords: Urine angiotensinogen; 24-Hour urine-sodium excretion; Hypertension; Chronic kidney disease 


\section{INTRODUCTION}

Increased renin-angiotensin system (RAS) activity is an independent risk factor for hypertension and when it is accompanied by sodium retention following volume expansion, the risk intensifies. In general, as a consequence of enhanced sodium retention, the activity of the systemic RAS is suppressed $[1,2]$. The question of whether salt intake leads to hypertension directly has been controversial for decades. Extensive studies have been conducted to identify the pathophysiological mechanisms responsible for the heterogeneity of responses due to increased salt intake [3-5]. Inappropriate or paradoxical activation of the intra-renal RAS, oxidative stress, and inflammatory cytokines in the kidney are key factors that lead to the development of salt - sensitive hypertension $[6,7]$. The intra-renal RAS, independent of the systemic RAS, plays an important role in the pathophysiology of hypertension and renal injury [6,8]. Moreover, high sodium diet modulates renal hemodynamics and tubular resorptive function to enhance sodium retention, volume expansion, and the development of hypertension [9]. Hypertension is associated with both increased intra-renal RAS activity and volume expansion [10], and it gets worse when these exist concomitantly.

Angiotensinogen (AGT) is the only known substrate for renin, the rate-limiting enzyme of the RAS, and changes in AGT levels as well as renin levels can control the activity of the RAS [11]. Angiotensin II in renal tissues is locally generated from AGT produced by the proximal tubule cells and delivered in the kidney. Urinary angiotensinogen (UAGT) may serve as a new biomarker to reflect the activity of intra-renal RAS activity, because it is derived largely from proximal tubular AGT. In addition, urinary excretion of AGT highly correlated with intra-renal AGT and angiotensin II levels [12-19].

The objective of the present study was to investigate whether UAGT excretion was associated with elevated blood pressure in chronic kidney disease (CKD) patients, and to evaluate the relationship between blood pressure, intra-renal RAS activity, and dietary sodium in patients with CKD.

\section{METHODS}

\section{Study subjects}

The design of the Korean Cohort Study for Outcome in Patients with Chronic Kidney Disease (KNOW-CKD) study has been described in detail in a previous study [20]. KNOW-CKD is a patient-based multicenter cohort study involving nine tertiary-care general hospitals in Korea. The KNOW-CKD enrolled ethnic Korean patients with CKD of stages 1 to 5 aged between 20 and 75 years. A total of 2,238 adults with CKD were enrolled from all participating centers between 2011 and 2015 in an ongoing prospective cohort study in collaboration with a multicenter cohort study. Of the total 2,238 patients, 1,955 patients who completed the measurements of 24-hour urinary sodium (24-hour UNa) excretion were included our analysis. The estimated glomerular filtration rate (eGFR) was calculated using the CKD Epidemiology Collaboration (CKD-EPI) creatinine (Cr) equation [21]. The serum and urine samples were subsequently collected on a regular basis according to the standardized protocol. All data, including clinical information, laboratory results, and outcomes were entered into a web-based, electronic, case-reporting form. The patients were divided into three groups according to their 24-hour UNa and UAGT to $\mathrm{Cr}(\mathrm{UAGT} / \mathrm{Cr}$ ) ratio values.

\section{Clinical data and laboratory analyses}

The clinical data, including detailed demographic information and baseline laboratory results, were extracted from the electronic data management system (PhactaX, Seoul, Korea). The morning spot urine samples were collected from all patients to measure the UAGT, urinary $\mathrm{Cr}$, albumin, and protein levels. The urine samples were centrifuged at $1,500 \mathrm{rpm}$ for 10 minutes at $4^{\circ} \mathrm{C}$. The urinary supernatants were pooled, and the UAGT concentrations were measured using human AGT ELISA kits (IBL, Takasaki, Japan). Intra-assay and inter-assay coefficients of variation for the human AGT ELISA kits were $4.4 \%$ and $4.3 \%$, respectively [22]. The urinary concentrations of AGT and albumin were normalized to the urinary $\mathrm{Cr}$ level. The resting blood pressure was measured at each clinic using an electronic sphygmomanometer. Based on the etiology of CKD, the patients were categorized as having glomerulonephritis, diabetic nephropathy, hypertensive nephropathy, and polycystic kidney disease. 


\section{Statistical analysis}

R language version 3.3.3 (R Foundation for Statistical Computing, Vienna, Austria) and T\&F program ver. 2.0 (YooJin BioSoft, Goyang, Korea) were used for all statistical analyses. The data were expressed as mean \pm $\mathrm{SD}$ for continuous variables. The continuous variables, including systolic blood pressure (SBP), diastolic blood pressure (DBP), 24-hour UNa, and UAGT/Cr were transformed to categorical variables having three subgroups corresponding to the three respective tertile ranges. For the continuous variables, Pearson correlation and Spearman correlation coefficient were computed. Linear regression analysis was performed to analyze the effect of each clinical measurement on the continuous response of SBP. Multivariable linear regression analysis was performed using backward stepwise procedure as a variable selection method to minimize Akaike information criterion (AIC). Significance level of o.1 was set for the univariable analysis to select initial input variables for the multivariable analysis (Supplementary Table 1).

Binary logistic regression analysis was performed to analyze the effect of each clinical measurement on the binary response of SBP (1st and 2nd group vs. 3rd group). For analyzing the combined effect of more than two variables on the SBP (1st and 2nd group vs. 3rd group), multivariable logistic regression analysis was performed using backward stepwise procedure as a variable selection method to minimize AIC. Significance level of 0.1 was set for the univariable analysis to select the initial input variables for the multivariable analysis.

\section{Ethical approval and informed consent}

The study protocol was approved by the Institutional Review Board at each participating clinical center-i.e., Seoul National University Hospital (1104-089-359), Seoul National University Bundang Hospital (B-1106/129oo8), Yonsei University Severance Hospital (4-20110163), Kangbuk Samsung Medical Center (2011-01-076), Seoul St. Mary's Hospital (KCı1OIMIo441), Gil Hospital (GIRBA2553), Eulji General Hospital (201105-01), Chonnam National University Hospital (CNUH-2011-092), and Inje University Pusan Paik Hospital (11-091) in 2011. Written informed consent was obtained from all study participants. This study was conducted in accordance with the principles of the Declaration of Helsinki.

\section{RESULTS}

\section{Baseline characteristics and comparison of study participants according to 24-hour UNa excretion}

A total of 1,955 patients (mean age, $48.7 \pm 12.4$ years; $60.7 \%$ male population) were included in this study. The etiology of primary renal disease in the subjects was hypertension in $18.4 \%$, autosomal dominant polycystic kidney disease in $17.3 \%$, diabetes in $23.6 \%$, and glomerulonephritis in $34.4 \%$ of the subjects. The mean 24 -hour UNa level was $158.33 \pm 70.10 \mathrm{mEq} /$ day. Table 1 represents the demographics and clinical features in the three 24-hour $\mathrm{UNa}$ categories. In the group with high 24-hour UNa excretion, the proportion of males, body mass index, waist-to-hip ratio, SBP, and DBP was higher. In addition, in the group with high 24-hour UNa, RAS blockers (angiotensin-converting enzyme inhibitors and angiotensin II receptor blocker) were more used, and serum hemoglobin and fasting blood glucose were also higher. On the other hand, mean UAGT/Cr was 20.03, 18.52, and $20.39 \mu \mathrm{g} / \mathrm{g}$ respectively, in the three groups and there were no significant differences among them. There were no pertinent differences in values of serum albumin, sodium, potassium concentration and urinary albumin $\mathrm{Cr}$ ratio. The 24-hour UNa had no correlation with logarithm (Log) of UAGT/Cr (coefficient $R$, o.005; $p$ $=0.831$; not shown $)$.

\section{Factors affecting SBP}

We evaluated factors affecting SBP in CKD patients. SBP was related to waist-to-hip ratio, 24-hour UNa, Log UAGT/Cr, eGFR < $15 \mathrm{~mL} / \mathrm{min} / 1.73 \mathrm{~m}^{2}$ (Table 2). SBP had a positive correlation with $\mathrm{DBP}, 24$-hour $\mathrm{UNa}$, and Log UAGT/Cr (Fig. 1).

\section{Effect of 24-hour UNa excretion and UAGT/Cr on SBP}

Fig. 1 represents the single regression analysis for SBP. SBP revealed a positive association with $\mathrm{DBP}$ (coefficient $R$, 0.633; $p<0.001$ ), Log UAGT/Cr (coefficient $R$, o.1; $p$ $<0.001$ ), and 24-hour UNa levels (coefficient $R$, 0.088; $p$ $<0.001$ ). Patients were divided into nine groups based on the third tertile of 24 -hour UNa and UAGT/Cr. Table 3 showed that in the three groups with the lowest 24-hour UNa (1st tertile), SBP increased as the UAGT/Cr increased. Similarly, in the three groups with the lowest 
Table 1. Baseline characteristics and clinical features of the patients in the different tertile groups based on the 24 -hour UNa excretion level

\begin{tabular}{|c|c|c|c|c|c|}
\hline \multirow{2}{*}{ Variable } & \multirow{2}{*}{ Total } & \multicolumn{3}{|c|}{ 24-hour UNa excretion } & \multirow{2}{*}{$p$ value } \\
\hline & & 1st tertile & 2nd tertile & 3rd tertile & \\
\hline Patient & $1,955(100)$ & $650(33 \cdot 3)$ & $653(33.4)$ & $652(33.4)$ & \\
\hline 24-hour UNa, mEq/day & $158.33 \pm 70.10$ & $87.55 \pm 26.44$ & $150.35 \pm 15.75$ & $236.91 \pm 50.52$ & $<0.001$ \\
\hline Male sex & $1,186(60.7)$ & $340(52.3)$ & $361(55 \cdot 3)$ & $485(74.4)$ & $<0.001$ \\
\hline Age, yr & $48.7 \pm 12.4$ & $49.3 \pm 12.7$ & $48.9 \pm 12.0$ & $48.0 \pm 12.2$ & 0.076 \\
\hline Etiology of CKD & & & & & 0.0015 \\
\hline Diabetic nephropathy & $462(23.6)$ & $157(24.2)$ & $152(23 \cdot 3)$ & $153(23 \cdot 5)$ & 0.926 \\
\hline Hypertensive nephropathy & $360(18.4)$ & $127(19 \cdot 5)$ & $107(16.4)$ & $126(19 \cdot 3)$ & 0.260 \\
\hline Glomerulonephritis & $673(34.4)$ & $208(32.0)$ & $226(34 \cdot 6)$ & $239(36.7)$ & 0.208 \\
\hline Polycystic kidney disease & $338(17 \cdot 3)$ & $122(18.8)$ & $132(20.2)$ & $84(12.9)$ & 0.001 \\
\hline eGFR category, $\mathrm{mL} / \mathrm{min} / 1.73 \mathrm{~m}^{2}$ & & & & & $<0.001$ \\
\hline$>90$ & $237(12.1)$ & $53(8.2)$ & $89(13.6)$ & $95(14.6)$ & \\
\hline $60-90$ & $364(18.6)$ & $102(15 \cdot 7)$ & $130(19.9)$ & $132(20.2)$ & \\
\hline $45-59$ & $353(18.1)$ & $123(18.9)$ & $102(15.6)$ & $128(19.6)$ & \\
\hline $30-44$ & $431(22.0)$ & $133(20.5)$ & $150(23.0)$ & $148(22.7)$ & \\
\hline $15-29$ & $449(23.0)$ & $182(28.0)$ & $149(22.8)$ & $118(18.1)$ & \\
\hline$<15$ & $121(6.2)$ & $57(8.8)$ & $33(5.1)$ & $31(4.8)$ & \\
\hline Body mass index, $\mathrm{kg} / \mathrm{m}^{2}$ & $24.57 \pm 3.40$ & $23.69 \pm 3.35$ & $24.56 \pm 3.28$ & $25 \cdot 54 \pm 3.34$ & $<0.001$ \\
\hline Waist-to-hip ratio, $\mathrm{m} / \mathrm{m}$ & $0.9 \pm 0.07$ & $0.89 \pm 0.07$ & $0.90 \pm 0.06$ & $0.91 \pm 0.06$ & $<0.001$ \\
\hline Systolic blood pressure, mmHg & $128.50 \pm 16.51$ & $127.22 \pm 16.98$ & $127.74 \pm 15.83$ & $130.99 \pm 16.53$ & $<0.001$ \\
\hline Diastolic blood pressure, $\mathrm{mmHg}$ & $76.85 \pm 11.17$ & $75.73 \pm 11.26$ & $76.42 \pm 11.01$ & $78.20 \pm 11.42$ & 0.002 \\
\hline $\mathrm{eGFR}, \mathrm{mL} / \mathrm{min} / 1.73 \mathrm{~m}^{2}$ & $50.41 \pm 30.17$ & $44.91 \pm 27 \cdot 37$ & $51.98 \pm 30.70$ & $54.93 \pm 30.69$ & $<0.001$ \\
\hline ACEIs or ARBs & $1,675(85.7)$ & $544(83.7)$ & $549(84.2)$ & $582(89 \cdot 3)$ & 0.006 \\
\hline UACR, g/g & $0.71 \pm 1.27$ & $0.55 \pm 0.98$ & $0.67 \pm 1.17$ & $0.80 \pm 1.51$ & 0.290 \\
\hline Hemoglobin, g/dL & $12.83 \pm 2.02$ & $12.49 \pm 1.92$ & $12.74 \pm 1.92$ & $13.35 \pm 2.08$ & $<0.001$ \\
\hline Fast blood glucose, mg/dL & $111.00 \pm 39.65$ & $108.32 \pm 36.89$ & $109.88 \pm 38.83$ & $114.70 \pm 42.00$ & $<0.001$ \\
\hline Albumin, mg/dL & $4.17 \pm 0.43$ & $4.19 \pm 0.39$ & $4.17 \pm 0.44$ & $4.21 \pm 0.43$ & 0.125 \\
\hline Sodium, mEq/L & $140.82 \pm 2.46$ & $140.82 \pm 2.41$ & $140.83 \pm 2.54$ & $140.84 \pm 2.44$ & 0.796 \\
\hline Potassium, mEq/L & $4.63 \pm 0.57$ & $4.64 \pm 0.58$ & $4.62 \pm 0.57$ & $4.64 \pm 0.56$ & 0.700 \\
\hline Uric acid, mg/dL & $7.02 \pm 1.92$ & $7.18 \pm 2.00$ & $6.85 \pm 1.87$ & $6.98 \pm 1.86$ & 0.008 \\
\hline UAGT/Cr, $\mu \mathrm{g} / \mathrm{g}$ & $19.40 \pm 42.28$ & $20.03 \pm 49.39$ & $18.52 \pm 35.34$ & $20.39 \pm 43.52$ & 0.835 \\
\hline Log UAGT/Cr, $\mu \mathrm{g} / \mathrm{g}$ & $0.75 \pm 0.68$ & $0.74 \pm 0.69$ & $0.76 \pm 0.66$ & $0.75 \pm 0.69$ & 0.871 \\
\hline
\end{tabular}

Values are presented as number (\%) or mean $\pm \mathrm{SD}$,

24-hour UNa, 24-hour urinary sodium; CKD, chronic kidney disease; eGFR estimated glomerular filtration rate; ACEI, angiotensin-converting-enzyme inhibitors; ARB, angiotensin receptor blocker; UACR, urine albumin-to-creatinine ratio; UAGT, urine angiotensinogen; Log, logarithm; Cr, creatinine.

UAGT/Cr values (1st tertile), SBP increased significantly as the 24-hour UNa increased. SBP was the highest (with a mean blood pressure of $131.873 \pm 1.384 \mathrm{mmHg}$ ) in the cluster with the highest tertiles (third groups) of both 24-hour UNa and UAGT/Cr compared to that of the other groups (Table 3).

After adjusting for clinical and basic demographic factors including age, sex, five stages according to the eGFR 
Table 2. Results of the multiple linear regression analysis using the backward stepwise method for systolic blood pressure

\begin{tabular}{lcr}
\hline Predictor & Regression coefficient $(95 \%$ CI $)$ & p value \\
\hline Age & $-0.004(-0.116$ to 0.108$)$ & 0.949 \\
Female & $0.168(-2.694$ to 3.030$)$ & 0.908 \\
Waist-to-hip ratio & $38.805(18.035$ to 59.575$)$ & 0.001 \\
24-hour UNa & $0.020(0.002$ to 0.038$)$ & 0.027 \\
Log UAGT/Cr & $1.950(0.103$ to 3.798$)$ & 0.039 \\
Log UACR & $1.577(-0.448$ to 3.602$)$ & 0.127 \\
eGFR category ${ }^{\text {a }}, \mathrm{mL} / \mathrm{min} / 1.73 \mathrm{~m}^{2}$ & & 0.285 \\
$\quad 60-90$ & $2.421(-2.013$ to 6.855$)$ & 0.916 \\
$30-59$ & $-0.237(-4.644$ to 4.170$)$ & 0.176 \\
$15-29$ & $3.599(-1.605$ to 8.803$)$ & 0.045 \\
$<15$ & $8.015(1.664$ to 14.367$)$ & \\
\hline
\end{tabular}

CI, confidence interval; 24-hour UNa, 24-hour urinary sodium; Log, logarithm; UAGT/Cr, urinary angiotensinogen-to-creatinine ratio; UACR, urinary albumin-to-creatinine ration; eGFR, estimated glomerular filtration rate.

${ }^{\mathrm{a}}$ Reference, $>90 \mathrm{~mL} / \mathrm{min} / 1.73 \mathrm{~m}^{2}$.
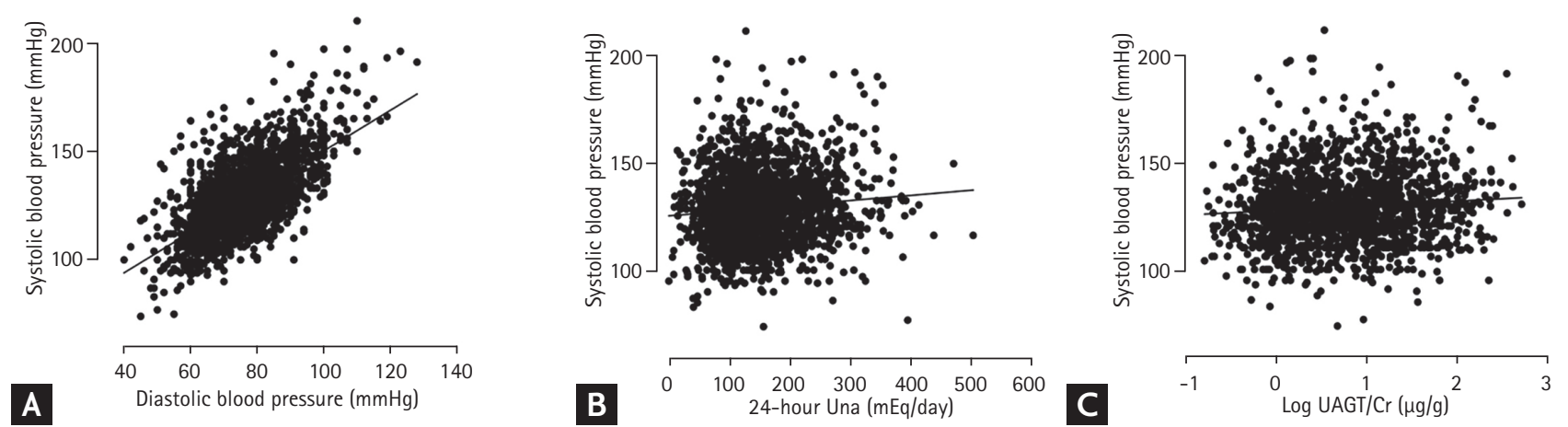

\begin{tabular}{lcc}
\hline Variable & Coefficient $R$ & $p$ value \\
\hline Diastolic blood pressure & 0.633 & $<0.001$ \\
24-hour Una & 0.1 & $<0.001$ \\
Log UAGT/Cr & 0.088 & $<0.001$ \\
\hline
\end{tabular}

Figure 1. Results of the single regression analysis for systolic blood pressure. Relationship between systolic blood pressure and (A) diastolic blood pressure, (B) 24-hour urinary sodium (24-hour UNa) excretion, and (C) logarithm of urine angiotensinogen-to-creatinine ratio (Log UAGT/Cr).

category, waist-to-hip ratio, body mass index, and hemoglobin level, the odds of having the 3rd tertile (highest) of SBP in each patient group was analyzed by multivariable binary logistic regression (Table 4). The odds ratio of the group with the lowest 24-hour UNa and UAGT/Cr was used as a reference. In this study, the risk of elevated SBP in the 3rd tertile of both the UAGT/Cr and 24-hour UNa groups was about 2.3 times higher than that in the reference group. High SBP was associated with elevated levels of both UAGT/Cr and 24-hour UNa.

In the three groups with the lowest 24-hour UNa (1st tertile), odds of an elevated SBP increased with a large difference as the UAGT/Cr increased. Similarly, in the three groups with the lowest UAGT/Cr values (1st tertile), odds of SBP increased considerably as the 24-hour UNa increased. Therefore, when the value of either 24- 
Table 3. Systolic blood pressure (mmHg) according to the 24-hour UNa levels (mEq/day) and UAGT/Cr $(\mu \mathrm{g} / \mathrm{g})$

\begin{tabular}{|c|c|c|c|c|}
\hline \multirow{2}{*}{ 24-hour $\mathrm{UNa}^{\mathrm{a}}$} & \multicolumn{3}{|c|}{$\mathrm{UAGT} / \mathrm{Cr}$} & \multirow{2}{*}{$p$ value } \\
\hline & 1st tertile & 2nd tertile & 3rd tertile & \\
\hline ist tertile & $125.269 \pm 1.384(n=169)$ & $129.885 \pm 1.388(n=170)$ & $129.077 \pm 1.392(n=168)$ & 0.049 \\
\hline 2nd tertile & $126.394 \pm 1.433(\mathrm{n}=164)$ & $130.09 \pm 1.347(n=181)$ & $129.293 \pm 1.378(n=169)$ & 0.110 \\
\hline 3rd tertile & $130.454 \pm 1.445(n=168)$ & $128.902 \pm 1.433(n=163)$ & $131.873 \pm 1.384(n=168)$ & 0.212 \\
\hline$p$ value & 0.041 & 0.882 & 0.204 & \\
\hline
\end{tabular}

Values are presented as mean $\pm \mathrm{SD}$ (n, sample number in each subgroup).

24-hour UNa, 24-hour urinary sodium; UAGT/Cr, urinary angiotensinogen-to-creatinine ratio.

${ }^{a} p=0.009 ; p$ value is computed using a post hoc algorithm of Tukey after performing one-way ANCOVA.

Table 4. OR (95\% CI) for the 3rd tertile (highest) of systolic blood pressure according to the 24-hour UNa and UAGT/Cr

\begin{tabular}{|c|c|c|c|}
\hline \multirow{2}{*}{ 24-hour UNa } & \multicolumn{3}{|c|}{ UAGT/Cr } \\
\hline & 1st tertile & 2nd tertile & 3rd tertile \\
\hline \multirow[t]{2}{*}{ 1st tertile } & 1 (reference) & $1.782(1.095-2.899)$ & $2.289(1.404-3.729)$ \\
\hline & & $p=0.02$ & $p \leq 0.001$ \\
\hline \multirow[t]{2}{*}{ 2nd tertile } & $1.313(0.798-2.158)$ & $1.715(1.060-2.777)$ & $1.667(1.021-2.724)$ \\
\hline & $p=0.283$ & $p=0.028$ & $p=0.041$ \\
\hline \multirow[t]{2}{*}{ 3rd tertile } & $2.121(1.298-3.466)$ & $1.564(0.953-2.567)$ & $2.395(1.478-3.879)$ \\
\hline & $p=0.003$ & $p=0.077$ & $p \leq 0.001$ \\
\hline
\end{tabular}

From multivariable binary logistic regression models stratified by $3 \times 3$ groups and adjusted for age, sex, 5 stages according to the estimated glomerular filtration rate category, waist-to-hip ratio, body mass index, and hemoglobin level (g/dL).

OR, odds ratio; CI, confidence interval; 24-hour UNa, 24-hour urinary sodium; UAGT/Cr, urinary angiotensinogen-to-creatinine ratio.

hour UNa or UAGT/Cr was low, SBP was more strongly affected by the other corresponding variable.

\section{DISCUSSION}

The present study demonstrated that enhanced intra-renal RAS activity and high urinary sodium excretion were associated with high SBP in CKD patients. It was conducted by using data from the KNOW-CKD study, which was a well-designed, cross-sectional study of 2,238 patients with CKD. The group with the highest tertile of UAGT/Cr and 24-hour UNa had the highest tertile SBP with an odds ratio of 2.395 compared to the rest of the group. The high salt intake and intra-renal RAS activity may be related to the group with the increased SBP.

Many observational studies have evaluated the relationship between high salt intake and blood pressure, and obtained conflicting results. Recently, however, numerous observational studies, Cochrane meta-analyses, and randomized trials have revealed that there is a close association between salt intake and elevated blood pressure [23-25]. Our study showed that for every $100 \mathrm{mEq} /$ day increase of 24-hour UNa, SBP increased by 2.0 $\mathrm{mmHg}(p=0.027)$ (Table 2). This is a small change compared to the results of the Korean National Health and Nutritional Examination Survey, which revealed that as 24 -hour UNa increased by $100 \mathrm{mEq} /$ day, SBP increased by $6.1 \mathrm{mmHg}$ [26]. This may be explained by the fact that sensitivity to changes in blood pressure due to sodium intake is very distinctive for an individual and is known to be associated with genetic susceptibility [27]. In salt-sensitive hypertensive patients, a long-term high-salt diet can cause hypertension and renal injury, more prominently than in the general population [2831]. When sodium retention occurs, the intra-renal RAS activity may be elevated, which may be the mechanism underlying salt-induced hypertension, which causes 
hypertensive renal injury [7]. Additionally, salt-sensitive patients exhibited higher blood pressure and less plasma renin activity suppression in response to elevated sodium intake [32-34]. In our present study, higher the salt intake, higher the SBP and DBP. This may affect CKD progression, even among those with better renal function and high eGFR. This is because high-salt diet is associated with increased renal injury and significant changes in the gene expression of renal cytokines that are related to the pro-inflammatory response and endothelial dysfunction, and attenuated cell differentiation and survival [35].

While previous studies conducted in patients classified as salt-sensitive groups have shown that high salt intake leads to an increase in intra-renal RAS activity, our study did not show a correlation between UAGT excretion and urinary sodium excretion. Understandably, as in our study we had not distinguished between salt-sensitive and salt-resistant groups. However, it showed that UAGT excretion was independently associated with SBP. It was also demonstrated that the group with the highest urinary sodium excretion and UAGT excretion showed the greatest odds of presenting with high SBP.

The strength of the present study is that it provides evidence in a relatively large, well-designed CKD patient cohort. To our knowledge, this was the first Asian study to show an effect of both UAGT and high-salt diet on SBP in CKD patients. Regardless, this study had several limitations. First, owing to the cross-sectional nature of the study, it was difficult to demonstrate the cause-effect relationship between SBP and 24-hour UNa and UAGT/Cr. Second, more than $85 \%$ of the patients with CKD were on RAS blocker; these patients could not be excluded or analyzed as a different group. Third, it does not provide insights on 24-hour UNa and daily sodium intake in CKD patients with impaired tubular function and sodium reabsorption.

In conclusion, both the intra-renal RAS and high-salt diet could be related to the elevation of SBP in CKD patients. Modulating and monitoring each of these in hypertensive CKD patients may help alleviate renal injury. While treating patients with hypertension, both the amount of sodium in the diet and the intra-renal RAS need to be considered. More research in the future will be needed to determine if dietary sodium restriction could be a more effective treatment for patients with increased intra-renal RAS activity.

\section{KEY MESSAGE}

1. Systolic blood pressure (SBP) showed a positive correlation with 24-hour urinary sodium (24hour UNa) and urine angiotensinogen-to-creatinine ratio (UAGT/Cr), in patients with chronic kidney disease (CKD).

2. SBP was the highest in the group with the highest 24-hour UNa (third tertile) and the group with the highest UAGT/Cr (third tertile).

3. These findings suggest that increased SBP in CKD patients is associated with both increased dietary sodium and intra-renal renin-angiotensin system activity.

\section{Conflict of interest}

No potential conflict of interest relevant to this article was reported.

\section{Acknowledgments}

This research was assisted by the Research Program funded by the Korea Centers for Disease Control and Prevention (2011E3300300, 2012E3301100, 2013E3301600,

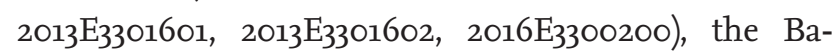
sic Science Research Program through the National Research Foundation of Korea (NRF) funded by the Ministry of Science, ICT \& Future Planning (2015R1DiA1Ao1061037), by the Bio \& Medical Technology Development Program of the NRF funded by the Korean government, MSIT (2017M3A9E8023001), and by a grant (BCR18015-1) of Chonnam National University Hospital Biomedical Research Institute.

\section{REFERENCES}

1. Griffing GT, Wilson TE, Melby JC. Alterations in aldosterone secretion and metabolism in low renin hypertension. J Clin Endocrinol Metab 1990;71:1454-1460.

2. Fisher ND, Hurwitz S, Ferri C, Jeunemaitre X, Hollenberg NK, Williams GH. Altered adrenal sensitivity to angiotensin II in low-renin essential hypertension. Hypertension 
1999;34:388-394.

3. Kopkan L, Hess A, Huskova Z, Cervenka L, Navar LG, Majid DS. High-salt intake enhances superoxide activity in eNOS knockout mice leading to the development of salt sensitivity. Am J Physiol Renal Physiol 2010;299:F656-F663.

4. Blaustein $\mathrm{MP}$, Leenen $\mathrm{FH}$, Chen $\mathrm{L}$, et al. How $\mathrm{NaCl}$ raises blood pressure: a new paradigm for the pathogenesis of salt-dependent hypertension. Am J Physiol Heart Circ Physiol 2012;302:H1031-H1O49.

5. Hauck C, Frishman WH. Systemic hypertension: the roles of salt, vascular $\mathrm{Na}+\mathrm{K}+$ AT'Pase and the endogenous glycosides, ouabain and marinobufagenin. Cardiol Rev 2012;20:130-138.

6. Kobori H, Nangaku M, Navar LG, Nishiyama A. The intrarenal renin-angiotensin system: from physiology to the pathobiology of hypertension and kidney disease. Pharmacol Rev 2007;59:251-287.

7. Majid DS, Prieto MC, Navar LG. Salt-sensitive hypertension: perspectives on intrarenal mechanisms. Curr Hypertens Rev 2015;11:38-48.

8. Navar LG, Kobori H, Prieto MC, Gonzalez-Villalobos RA. Intratubular renin-angiotensin system in hypertension. Hypertension 2011;57:355-362.

9. Weinberger MH. Sodium, potassium, and blood pressure. Am J Hypertens 1997;10(5 Pt 2):46S-48S.

10. Rodriguez-Iturbe B, Romero F, Johnson RJ. Pathophysiological mechanisms of salt-dependent hypertension. Am J Kidney Dis 2007;50:655-672.

11. Brasier AR, Li J. Mechanisms for inducible control of angiotensinogen gene transcription. Hypertension 1996;27(3 Pt 2):465-475.

12. Kobori H, Harrison-Bernard LM, Navar LG. Urinary excretion of angiotensinogen reflects intrarenal angiotensinogen production. Kidney Int 2002;61:579-585.

13. Kobori H, Nishiyama A, Abe Y, Navar LG. Enhancement of intrarenal angiotensinogen in Dahl salt-sensitive rats on high salt diet. Hypertension 2003;41:592-597.

14. Kobori H, Nishiyama A, Harrison-Bernard LM, Navar LG. Urinary angiotensinogen as an indicator of intrarenal angiotensin status in hypertension. Hypertension 2003;41:42-49.

15. Yamamoto T, Nakagawa T, Suzuki H, et al. Urinary angiotensinogen as a marker of intrarenal angiotensin II activity associated with deterioration of renal function in patients with chronic kidney disease. J Am Soc Nephrol
2007;18:1558-1565.

16. Urushihara M, Kondo S, Kagami S, Kobori H. Urinary angiotensinogen accurately reflects intrarenal Renin-Angiotensin system activity. Am J Nephrol 2010;31:318-325.

17. Nishiyama A, Konishi Y, Ohashi N, et al. Urinary angiotensinogen reflects the activity of intrarenal renin-angiotensin system in patients with IgA nephropathy. Nephrol Dial Transplant 2011;26:170-177.

18. Mills KT, Kobori H, Hamm LL, et al. Increased urinary excretion of angiotensinogen is associated with risk of chronic kidney disease. Nephrol Dial Transplant 2012;27:3176-3181.

19. Wu H, Liang Y, Zheng Y, et al. Up-regulation of intrarenal renin-agiotensin system contributes to renal damage in high-salt induced hypertension rats. Kidney Blood Press Res 2014;39:526-535.

20. Oh KH, Park SK, Park HC, et al. KNOW-CKD (KoreaN cohort study for Outcome in patients With Chronic Kidney Disease): design and methods. BMC Nephrol 2014;15:80.

21. Levey AS, Coresh J, Greene T, et al. Using standardized serum creatinine values in the modification of diet in renal disease study equation for estimating glomerular filtration rate. Ann Intern Med 2006;145:247-254.

22. Katsurada A, Hagiwara Y, Miyashita K, et al. Novel sandwich ELISA for human angiotensinogen. Am J Physiol Renal Physiol 2007;293:F956-F96o.

23. Aburto NJ, Ziolkovska A, Hooper L, Elliott P, Cappuccio FP, Meerpohl JJ. Effect of lower sodium intake on health: systematic review and meta-analyses. BMJ 2013;346:f1326.

24. He FJ, Li J, Macgregor GA. Effect of longer term modest salt reduction on blood pressure: cochrane systematic review and meta-analysis of randomized trials. BMJ 2013;346:fi325.

25. Cook NR, Appel LJ, Whelton PK. Sodium intake and allcause mortality over 20 years in the trials of hypertension prevention. J Am Coll Cardiol 2016;68:1609-1617.

26. Oh J, Lee J, Koo HS, Kim S, Chin HJ. Estimated 24-hour urine sodium excretion is correlated with blood pressure in Korean population: 2009-2011 Korean National Health and Nutritional Examination Survey. J Korean Med Sci 2014;29 Suppl 2(Suppl 2):S109-S116.

27. Sanada H, Jones JE, Jose PA. Genetics of salt-sensitive hypertension. Curr Hypertens Rep 2011;13:55-66.

28. Intersalt Cooperative Research Group. Intersalt: an international study of electrolyte excretion and blood pressure. Results for 24 hour urinary sodium and potassium 
excretion. BMJ 1988;297:319-328.

29. Cutler JA, Follmann D, Allender PS. Randomized trials of sodium reduction: an overview. Am J Clin Nutr 1997;65(2 Suppl):643S-651S.

30. He J, Ogden LG, Vupputuri S, Bazzano LA, Loria C, Whelton PK. Dietary sodium intake and subsequent risk of cardiovascular disease in overweight adults. JAMA 1999;282:2027-2034.

31. Sacks FM, Svetkey LP, Vollmer WM, et al. Effects on blood pressure of reduced dietary sodium and the Dietary Approaches to Stop Hypertension (DASH) diet. DASH-Sodium Collaborative Research Group. N Engl J Med 2001;344:3-10.

32. Giner V, Poch E, Bragulat E, et al. Renin-angiotensin sys- tem genetic polymorphisms and salt sensitivity in essential hypertension. Hypertension 2000;35(1 Pt 2):512-517.

33. Poch E, Gonzalez D, Giner V, Bragulat E, Coca A, de La Sierra A. Molecular basis of salt sensitivity in human hypertension. Evaluation of renin-angiotensin-aldosterone system gene polymorphisms. Hypertension 2001;38:12041209.

34. Hasimu B, Nakayama T, Mizutani Y, et al. Haplotype analysis of the human renin gene and essential hypertension. Hypertension 2003;41:308-312.

35. Gu JW, Bailey AP, Tan W, Shparago M, Young E. Longterm high salt diet causes hypertension and decreases renal expression of vascular endothelial growth factor in Sprague-Dawley rats. J Am Soc Hypertens 2008;2:275-285. 
Supplementary Table 1. Results of the univariable linear regression analysis using the systolic blood pressure as the response

\begin{tabular}{|c|c|c|c|}
\hline Predictor (response = systolic blood pressure) & $\begin{array}{l}\text { Coefficient } \\
(95 \% \mathrm{CI})\end{array}$ & $\begin{array}{l}\text { Standardized coefficient } \\
(95 \% \mathrm{CI})\end{array}$ & $p$ value \\
\hline Age & $0.071(0.015$ to 0.126$)$ & $0.053(0.011$ to 0.095$)$ & 0.013 \\
\hline Sex & & & $<0.001$ \\
\hline Male vs. Female & $3.131(1.727$ to 4.535$)$ & $0.092(0.051$ to 0.134$)$ & $<0.001$ \\
\hline eGFR category & $-0.061(-0.083$ to -0.038$)$ & $-0.111(-0.153$ to -0.07$)$ & $<0.001$ \\
\hline Body mass index & $0.67(0.469$ to 0.872$)$ & 0.138 (0.096 to 0.179) & $<0.001$ \\
\hline Hemoglobin & $-0.554(-0.896$ to -0.212$)$ & $-0.068(-0.11$ to -0.026$)$ & 0.002 \\
\hline Waist-to-hip ratio & $43.847(33.225$ to 54.469$)$ & $0.175(0.133$ to 0.218$)$ & $<0.001$ \\
\hline 24-hour UNa & $0.024(0.013$ to 0.034$)$ & $0.1(0.055$ to 0.144$)$ & $<0.001$ \\
\hline UACR & $3.105(1.822$ to 4.389$)$ & $0.153(0.09$ to 0.216$)$ & $<0.001$ \\
\hline Log UAGT/Cr & 2.141 (0.98 to 3.303$)$ & $0.088(0.04$ to 0.135$)$ & $<0.001$ \\
\hline ACEIs or ARBs & $2.076(0.122$ to 4.03$)$ & $0.044(0.003$ to 0.086$)$ & 0.037 \\
\hline eGFR category, $\mathrm{mL} / \mathrm{min} / 1.73 \mathrm{~m}^{2}(\%)$ & & & $<0.001$ \\
\hline $60-90$ vs. $>90$ & $1.394(-1.137$ to 3.925$)$ & $0.125(-0.102$ to 0.352$)$ & 0.28 \\
\hline $45-59$ vs. $>90$ & $1.452(-1.101$ to 4.006$)$ & $0.13(-0.099$ to 0.359$)$ & 0.265 \\
\hline $30-44$ vs. $>90$ & $2.033(-0.429$ to 4.495$)$ & $0.182(-0.038$ to 0.403$)$ & 0.106 \\
\hline $15-29$ vs. $>90$ & $4.131(1.699$ to 6.564$)$ & $0.37(0.152$ to 0.588$)$ & $<0.001$ \\
\hline$<15$ vs. $>90$ & $8.997(5.652$ to 12.341$)$ & $0.806(0.506$ to 1.106$)$ & $<0.001$ \\
\hline eGFR category (two groups), $\mathrm{mL} / \mathrm{min} / 1.73 \mathrm{~m}^{2}(\%)$ & & & 0.009 \\
\hline$\leq 90$ vs. $>90$ & 2.841 (0.719 to 4.963$)$ & $0.056(0.014$ to 0.097$)$ & 0.009 \\
\hline Etiology of CKD & & & $<0.001$ \\
\hline Diabetic nephropathy & 4.787 (1.748 to 7.825$)$ & $0.376(0.137$ to 0.615$)$ & 0.002 \\
\hline Hypertensive nephropathy & $-2.015(-5.136$ to 1.107$)$ & $-0.158(-0.404$ to 0.087$)$ & 0.206 \\
\hline Glomerulonephritis & $-5.861(-8.782$ to -2.94$)$ & $-0.461(-0.69$ to -0.231$)$ & $<0.001$ \\
\hline Polycystic kidney disease & $-1.308(-4.475$ to 1.859$)$ & $-0.103(-0.352$ to 0.146$)$ & 0.418 \\
\hline
\end{tabular}

CI, confidence interval; eGFR, estimated glomerular filtration rate; 24-hour UNa, 24-hour urinary sodium; UACR, urine albumin-to-creatinine ratio; Log, logarithm; UAGT, urine angiotensinogen; ACEI, angiotensin-converting-enzyme inhibitor; ARB, angiotensin receptor blocker; CKD chronic kidney disease. 